\title{
Simulation and Analysis of a Combined Mobility Model with Obstacles
}

\author{
Emiliano Pecchia \\ University of Pisa \\ Telecommunication Engineering \\ 56126 Pisa, Italy \\ +393471756700 \\ epshouse@gmail.com
}

\author{
David Erman \\ Blekinge Institute of Technology \\ Campus Gräsvik, \\ 37179 Karlskrona, Sweden \\ +46455385658 \\ david.erman@bth.se
}

\author{
Adrian Popescu \\ Blekinge Institute of Technology \\ Campus Gräsvik, \\ 37179 Karlskrona, Sweden \\ +46455385659 \\ adrian.popescu@bth.se
}

\begin{abstract}
The use of simulations has become increasingly frequent in the study and the performance evaluation of network systems. The simulation environment deeply influences the behaviour of results, so a model that simulates a realistic movement of the nodes is necessary for the study of wireless networks. Simple mobility models do not provide realistic scenarios. Often movements are completely random, uncorrelated and in open space, without the chance of considering the effects of obstacles or rules that limit and guide the movement.

In this paper, we propose a more realistic mobility model, studied for indoor environments (but applicable to outdoor models as well). Given the map of the obstacles in the simulation area (e.g. a floor plan), the nodes have the possibility to move in random walk just avoiding to cross the obstacles (e.g. walls), or following a specified virtual path that connects all the simulation area, or a hybrid of the two. Our tool creates a file containing the movement of the nodes during the whole simulation time. Simulation results show that nodes are highly dependent on the different obstacles maps and pathways. Furthermore, a mathematical demonstration is given to validate the results obtained by simulation in a simple case.
\end{abstract}

Categories and Subject Descriptors: C.2.1 [Wireless communication]

General Terms: Algorithms, Performance

Keywords

Simulation, Mobility Model, Wireless Network, NS-2

Permission to make digital or hard copies of all or part of this work for personal or classroom use is granted without fee provided that copies are not made or distributed for profit or commercial advantage and that copies bear this notice and the full citation on the first page. To copy otherwise, or republish, to post on servers or to redistribute to lists, requires prior specific permission and/or a fee.

Simutools'09, March 2-6, 2009, Rome, Italy.

Copyright 2004 ACM 1-58113-000-0/00/0004 ...\$5.00.

\section{INTRODUCTION}

Simulation modelling is an important tool for the study and evaluation of the behaviour of wireless networks. The use of simulations provides several benefits, e.g., compared to real tests, it allows a easier what-if evaluation, with the possibility of easy choice and change of parameters. Furthermore, simulations provide a compression/expansion of time for a more detailed analysis of the behaviour of the system. These and other factors have contributed to simulations becoming a very powerful tool for the study of networks and the development of new technologies.

In the evaluation of wireless networks, simulation is strongly influenced by the mobility model chosen, as shown in [2], [3],[13]. In the real world, the movement of a node is influenced by several reasons. Usually it has to face the presence of obstacles, and the destination is typically not random. So the node decides its path thinking about avoiding obstacles and, usually, making the shortest way to the chosen destination. For instance, in indoor environment, when a node wants to move from a room to another, it just follows the shortest path to the chosen room along the corridors without stopping. In simulation, unrealistic and too simplified models will likely produce unrealistic results. A number of mobility models have been already proposed. Some of these create a random uncorrelated movement in a simulation area without obstacles. They are not accurate, but usually they are used because they are easy to implement and yield shorter simulation times. Some other models consider the presence of obstacles and the use of pathways. They are more accurate, but also more complex and they take more time to run.

In this paper we propose an improvement of a mobility model [4] that offers the chance to incorporate obstacles and pathways in the simulation area in order to create more realistic movements. Our model loads the map of the obstacles from an image file and a virtual pathway graph file. Then it is possible for the user to decide if the nodes: a) have to move according to a random walk, b) along the pathway, or c) in a hybrid movement. In the first case, the movement is the same as the Random Waypoint Model (RWP)[14], but nodes have to avoid crossing the obstacles. In the second case, the nodes choose a destination vertex in the pathway graph, and they move to that location using the shortest path. In the third case, the nodes use the RWP, but sometimes move to different areas using the pathway graph. Then they start moving in RWP again.

The presence of obstacles affects not only the movement of the nodes, but also the signal propagation. In our model the 
attenuation of a signal between source and destination depends not only on the distance of the two nodes, but also on the material properties of the obstacles placed in between.

To evaluate our mobility model we used the parameters defined in [5]. The results of two of these parameters are also validated by mathematical analysis, studying a very simple case.

The rest of the paper is organized as follows: Section 2 shows briefly the main mobility models already existing, explaining advantages and drawbacks. Section 3 describes the mobility model introduced in [4], and motivates our choice of improving it, while Section 4 explains in details our version of the model. Section 5 shows the evaluation of our mobility model, studying the results of the simulation. Section 6 is dedicated to a mathematical demonstration of some of those results, and Section 7 presents the conclusions of the paper.

\section{RELATED WORK}

A variety of mobility models have been proposed during the years for the study of wireless networks. The Random Walk mobility model was introduced by Einstein in 1926, and models the movement of each node from the current location to a new one by randomly choosing direction and speed, from a predefined range $[0,2 \pi]$ and [Vmin, $V \max ]$ respectively. After a certain interval of time (or a chosen distance travelled), the node calculates a new direction and speed. If the node reaches the boundary of the simulation area, it will be absorbed, bounced off, or wrapped around to the other side of the area. This behaviour results in sudden stops and sharp turns, so it can rarely describe a realistic movement. However, other more complicated mobility models, like the one proposed in [6], make use of the Random Walk because of its simplicity. Another simple model is the Random Waypoint (RWP) [14]. In this model the node alternates periods of movement and pause. It randomly chooses a destination point and moves to it with a constant speed. The speed is uniformly chosen in [Vmin, Vmax]. When the node reaches the destination point, it pauses for a random time and then calculates a new destination and speed. Again, this model can rarely describe a realistic movement. Besides, the density of the nodes inside the simulation area is not uniform, as proved in [7]. In fact, the nodes tend to gather in the centre of the area. This problem is not present in the Random Direction model [15], in which the node moves with a random speed and direction until it reaches the boundary of the simulation area. It then selects a new speed and moves in a randomly chosen direction until it reaches the boundary again. The City Section, or Manhattan [2], model provides realistic movements for a section of a city, where the movement of the nodes are affected by geographical (predefined paths caused by obstacles) and guidelines (traffic laws) factors. In this model the node starts at a defined point at some street and chooses a destination point. Then it travels towards it respecting the traffic laws (one way streets, speed limits, etc.). The model can be improved including pauses at the intersections (for instance simulating traffic lights), acceleration/deceleration, and variation of concentration in certain sectors (rush hour) and so on [8]. Another model for outdoor environments is the Obstacle Model proposed in [9]. It allows the placement of obstacles in the simulation area, which affects the movement of the nodes and the signal propagation. Once the obstacles (that simulate the presence of buildings in the area) are placed, the model creates a Voronoi diagram [10] using the vertex of the buildings. The nodes will move on this diagram, choosing destination points and reaching them moving across the shortest path. This model introduces the possibility to add obstacles in the simulation area, but it is good just for outdoor environment and also the movement of the nodes is restricted to the Voronoi graph created. It rarely can simulate an indoor environment. In [11] the 3-D Building model was proposed. It allows emulating the behaviour of nodes inside a multi-floor building. The node can move horizontally on a floor bounded by outer walls. It can choose to go forward, back, left or right with a certain probability and moving with a random speed uniformly distributed in $[0, \mathrm{Vmax}]$. The node can also move vertically, but only if it is situated in a special region of the floor, called "elevator region". It then selects a destination floor and move to it. If the node is in the basement, it can also exit from the building.

\section{ORIGINAL MODEL}

The model proposed in [4] allows emulating the movement of nodes in the simulation area in presence of obstacles. The original project of this model is divided in two different sub-models.

In the first one, called Constrained Mobility (CM), the obstacles affect only the propagation, not the movement. The model reads the map of the obstacles from an image file, in which every colour correspond to a different material, with different properties. The relations between colour and properties are written in a second file. Then it reads a graph (given by the user) from another file. The nodes will move along this graph, selecting as destination a random vertex of the graph and moving there choosing the path with the minimum number of hops. It is possible to select which vertices of the graph are possible destination vertices, using the other ones just to link the paths between them.

In the second sub-model, called the Shell Model, the nodes move following the rules of the RWP model, just avoiding crossing the external perimeter of the area and, then, ignoring the internal obstacles of the map, which affect just the propagation. In this sub-model the graph file is not read.

The structure of this original model allows creating a very complex and detailed map of the obstacles, but it can barely represent a realistic movement in an indoor environment. The nodes can or just move in a prefixed path, or move in a completely random way. It is not realistic that a node enters into a room and remains steady at one point. On the other side, moving randomly, the internal obstacles are completely ignored and, at every step, the movement is not correlated with the previous ones.

In the CM model there is also another problem. The path calculated for reaching the destination vertex can, depending on the graph, not be the shortest (usually the one most used in the real world).

\section{OUR MODEL}

All the problems presented in the previous section have been fixed in our model. First, we improved the Shell Model enabling the effects of the internal obstacles even for the nodes movement and not only for the signal propagation. To do this, we needed to define crossable obstacles, i.e., areas of the map (like doors) that 
don't stop the movement of the nodes, but attenuate the propagation. Despite this enhancement, the new model is not realistic either. In fact, moving randomly in an indoor scenario, the chance of leaving a room is low, so the probability of reaching rooms far from the initial one is very low. The idea is then to use both the CM and the new Shell models together, creating a new hybrid model. The nodes can switch from one sub-model to another, creating a more realistic behaviour. At every step they can move in RWP model with a probability $r w$, or they can jump into the graph and move to a destination vertex following the shortest pathway (calculated by the Dijkstra's algorithm [12]) with a probability $1-r w$. Note that for $r w=1$ and $r w=0$ our model returns to the original one. In practice $r w$ is the parameter that measures the distance of our model from the two sub-models. For a generic $r w$, the nodes usually move randomly in a room and use the graph to go into another room. Leaving the room is also possible during the RWP movement. In fact the node chooses a random destination in line of sight, and it can be a point belonging to another room. Furthermore, it is also possible to assign different values to the parameter $r w$ for different sub-areas of the simulation. It allows, for example, distinguishing the behaviour of the nodes in rooms (where the RWP model fits better) and in corridors (where the use of the graph is more suitable). A random speed uniformly distributed between [Vmin, $V \max ]$ is chosen for every RWP movement and at every vertex of the path reached. It is possible to choose the use or not of the pause. With the pause, the node stops for a random time uniformly distributed between $[0, \mathrm{~T}]$ at the end of every RWP movement and every time it reaches the destination vertex when it uses the graph.

In order to fix the problems of the original version of the model, and to provide these new features, our model has been designed as follows. The image that contains the map of the obstacles becomes more elaborated, since every sub-area has to be painted with a different colour. In Figure 1 there is a simple example of floor plan, with the related graph.

Note that the colour of the entrances is different from the one of the walls. There are two reasons: the door has different material properties from the walls. Also the door is not a real movement obstacle, it just affects the propagation. Note also that the graph must ensure that every sub-area has a destination vertex inside, in order to be used by the nodes as a reference point for jumping into the graph. When a node decides to use the graph, it first checks whether it is in line of sight with the destination vertex associated to the sub-area it is in. If it is not, the node continues moving in RWP.

\section{SIMULATIONS}

The main objective of this evaluation is to understand how the parameter $r w$ and the use of different maps influence the results of the simulation.

\subsection{Metrics}

The metrics evaluated are some of the ones suggested in [5] for the study of ad-hoc networks, and they are:

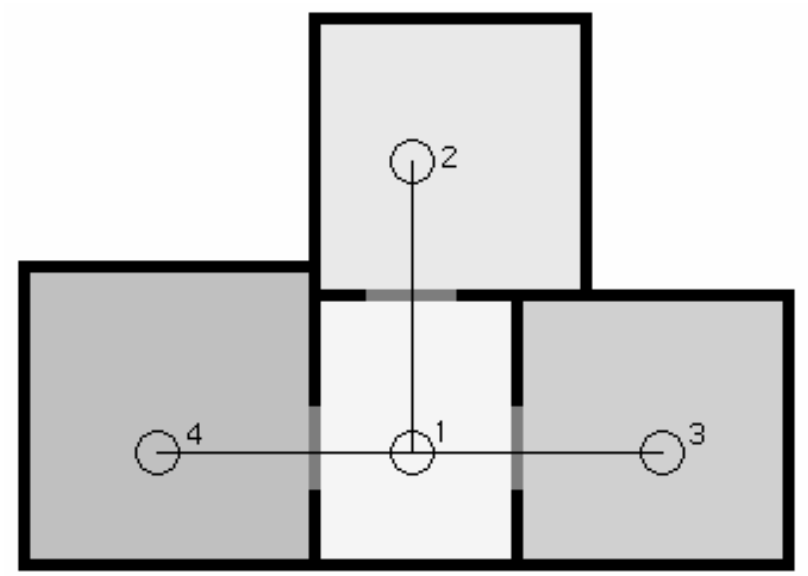

\begin{tabular}{|c|c|c|c|}
\cline { 3 - 4 } \multicolumn{2}{c|}{} & $\begin{array}{c}\text { Propagation } \\
\text { Obstacle }\end{array}$ & $\begin{array}{c}\text { Movement } \\
\text { Obstacle }\end{array}$ \\
\hline \multirow{2}{*nyy}{} & Sub-area 1 & - & - \\
\hline & Sub-area 2 & - & - \\
\hline & Sub-area 3 & - & - \\
\hline & Sub-area 4 & - & - \\
\hline & Doors & X & X \\
\hline
\end{tabular}

Figure 1 - Example of map with the associated graph

- Link Changes: Number of transitions between "off" and "on" status for a pair of nodes. It counts the number of times the link between two nodes is established.

- Link Duration: Duration of link in status "on".

- $\quad$ Node Degree: Number of neighbours per node. Relative Speed:

$$
R S(i, j, t) \triangleq\left|\overrightarrow{v_{i}}(t)-\overrightarrow{v_{j}}(t)\right|
$$

where $\overrightarrow{v_{i}}(t)$ is the velocity vector of the node $i$ at the time $t$. The relative speed is calculated only if the distance between the nodes $i$ and $j$ at the time $t$ is $D_{i, j}(t) \leq c \cdot R$, where $c$ is a constant and $R$ is the transmission range of the nodes. In our evaluation $c=2$.

- Spatial Correlation:

$$
D_{\text {spat }}(i, j, t) \triangleq R D\left(\overrightarrow{v_{i}}(t), \overrightarrow{v_{j}}(t)\right) \cdot S R\left(\overrightarrow{v_{i}}(t), \overrightarrow{v_{j}}(t)\right)
$$

where RD is the relative direction between two vectors, and it is defined as

$$
R D(\vec{a}, \vec{b}) \triangleq \frac{\vec{a} \cdot \vec{b}}{|\vec{a}| \cdot|\vec{b}|}
$$

and SR is the speed ratio between two vectors, defined as 


$$
S R(\vec{a}, \vec{b}) \triangleq \frac{\min \{|\vec{a}|,|\vec{b}|\}}{\max \{|\vec{a}|,|\vec{b}|\}}
$$

As the relative speed, the spatial correlation is calculated only if the distance between the nodes $i$ and $j$ at the time $t$ is $D_{i, j}(t) \leq c \cdot R$, with $c=2$.

- Temporal Correlation:

$$
D_{\text {temp }}\left(i, t, t^{\prime}\right) \triangleq R D\left(\overrightarrow{v_{i}}(t), \overrightarrow{v_{i}}\left(t^{\prime}\right)\right) \cdot \operatorname{SR}\left(\overrightarrow{v_{i}}(t), \overrightarrow{v_{i}}\left(t^{\prime}\right)\right)
$$

the temporal correlation is calculated only if the difference between $t$ and $t$ ' is less than a pre-fixed value ( 5 seconds in our evaluation).

The previous metrics has been extracted during the simulation and then calculated as the average over node pairs and time instants.

The evaluation of protocol performance metrics is not considered in this paper.

\subsection{Simulation Environment}

Our tool creates files compatible with the input mobility files used by NS2. We have run the tool using 3 different maps. The first one is a floor plan of a building with a big number of pretty small rooms and long corridors (Figure 2). This map, taken from the original model [4], can easily represent places like offices, hotels, hospitals etc. The second one is a simpler floor plan with few, but very wide rooms (Figure 3). Places like schools and malls can be represented by this map. The third map shows that this model can be also easily used to study outdoor environment or huge indoor areas like stations, airports, etc (Figure 4). An additional scenario has been simulated to study the simplest case possible: a squared area without any obstacles inside and a single destination vertex positioned in the centre. This map has been used to study and mathematically analyze two of the results obtained (Section 6).

The simulations run for a period of 1800 (simulated) seconds, in which 30 nodes move in an area of $70 \mathrm{~m} \times 70 \mathrm{~m}$ (total) with a random speed uniformly distributed in $[1,5] \mathrm{m} / \mathrm{s}$. The pause time (when used) has a uniform distribution in $[0,180]$ seconds. The value of parameter $r w$ has been chosen for the values between 0 and 1 , with step 0.1 . At the beginning of the simulations the nodes are randomly distributed on the destination vertices of the graph. The maximum node transmission range is $25 \mathrm{~m}$. Every simulation has been run for 30 times.

\subsection{Results}

All the metrics are calculated in function of the parameter rw, and the both cases of the use or not of the pause are shown. The confidence intervals of the results are not plotted because they are very narrow in all the graphs.

The average number of link changes throughout the simulations is shown in Figure 5. When $r w$ is close to 0 the results are influenced mostly by the pathway graph. This value is higher for the map 2, where the graph is less dense.

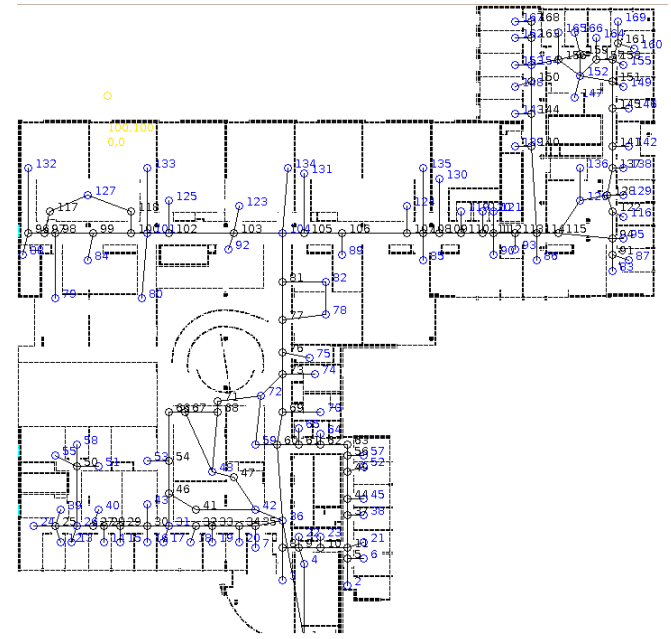

Figure 2 - Map 1

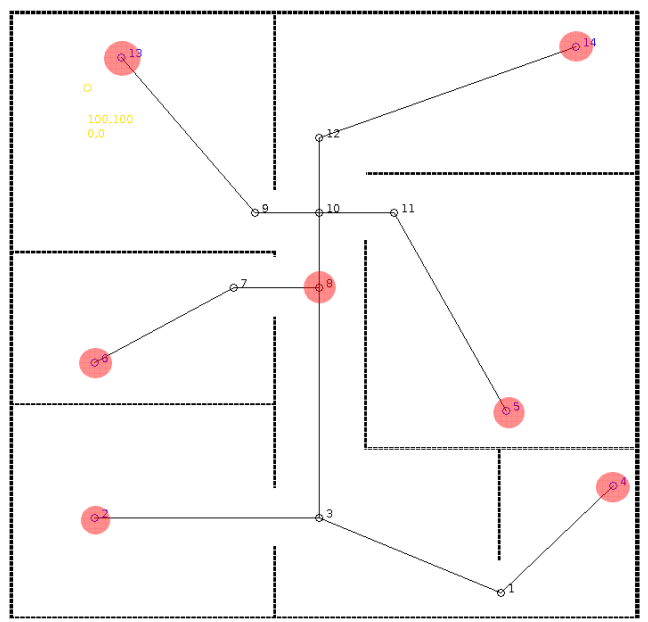

Figure 3 - Map 2

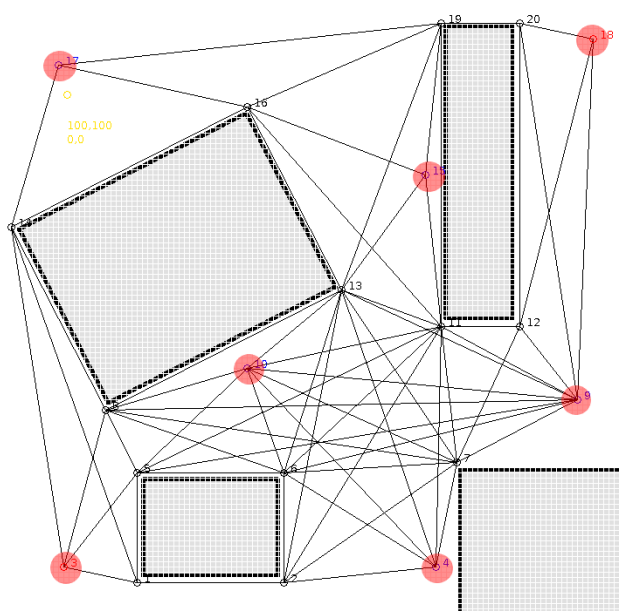

Figure 4 - Map 3

For that reason, the nodes moving along the graph choose every time a destination vertex far from the initial one and then move for long distances. This causes a higher number of link 
establishments and interruptions. When $r w$ is close to 1 the nodes move mostly in RWP, so the results depend mostly on the shape of the floor plan. The chance to remain in a small room is less than the one of remaining in a big one, so the nodes tend to move to large areas. In map 2 and 3 , the entire simulation area is composed by wide sub-areas, in which the nodes tend to distribute uniformly. In map 1 the nodes instead tend move to the long corridors, and they start moving for longer distances. So the number of link changes gets higher. When the pause is used, the global mobility is reduced and the average number of link changes goes down. When $r w$ is high, the number of pauses increases, and the effect is bigger.

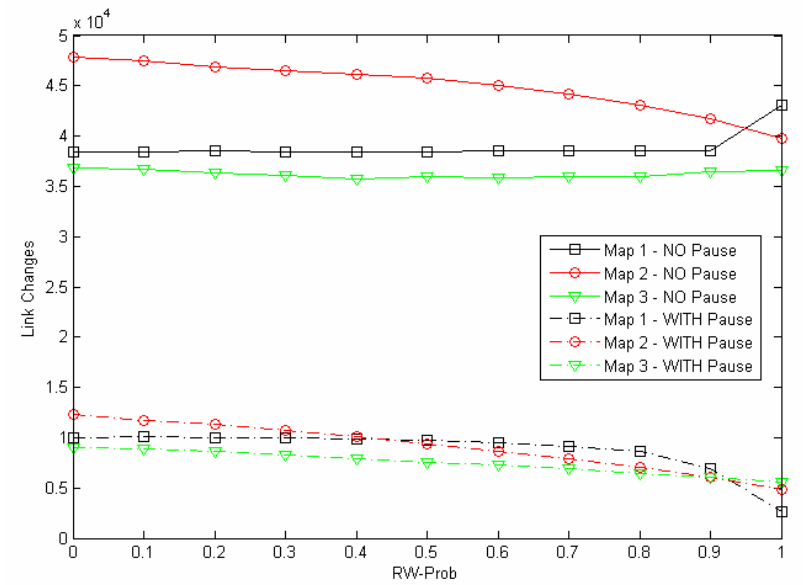

Figure 5 - Average number of link changes

Figure 6 shows the average link duration graph. The value in map 1 is higher because the graph is denser and it is more probable for a node to choose a close destination and to avoid the interruption of a link. Clearly, the use of the pause makes the link durations longer.

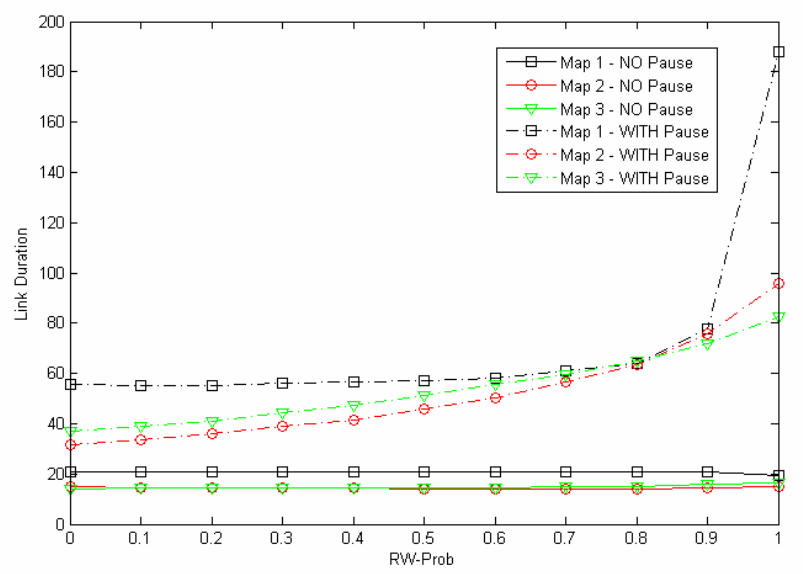

Figure 6 - Average link duration
The average node degree depends strongly on the shape of the pathway graphs (see Figure 7). A denser graph brings the node to be closer, with a higher average number of neighbours. Increasing $r w$ causes three different effects: the node degree does not change for map 1; it increases for map 2 and decreases for map 3. The presence of the pause forces the nodes to be more scattered on the simulation area, especially for graphs with less dense destination vertices, decreasing the node degree.

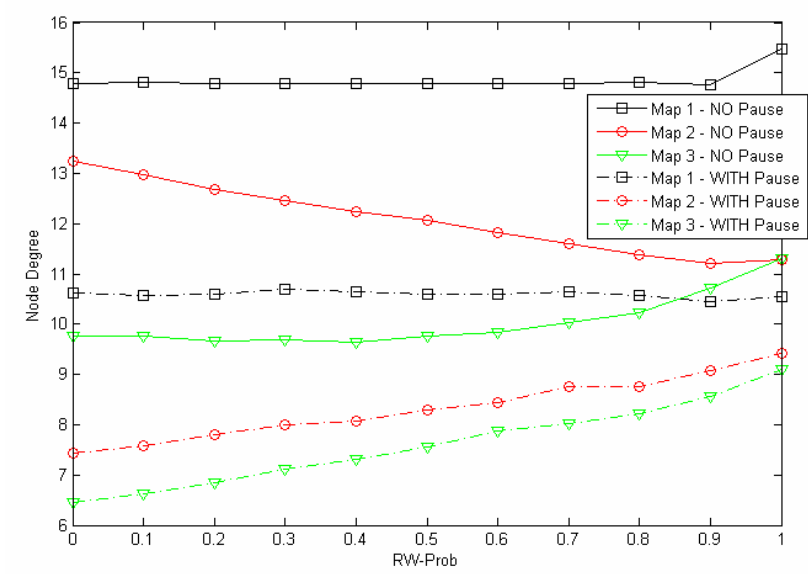

Figure 7 - Average node degree

Relative speed and spatial correlation turned out not to be relevant parameters, because the results did not depend on neither the map nor the parameter $r w$.

The average temporal correlation is shown in Figure 8. To explain the results it is necessary to analyze the two parameters that influence the temporal correlation: relative direction and speed ratio.

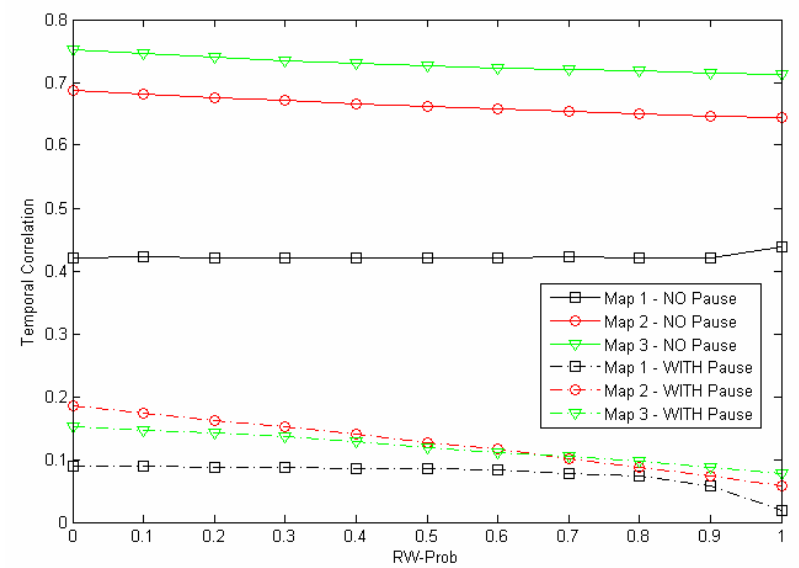

Figure 8 - Average temporal correlation

When $r w$ is low, the relative direction depends mostly on the shape of the pathways graphs (Figure 9). Smooth paths give 
higher RD than paths with sharp curves. Even if the graph of map 3 is very complex, it allows the nodes to make very smooth paths. In the graph of map 2 the changes of direction are sharp, but the interconnection veritces are very few, so the nodes move mostly in a straight direction. In the graph of map 1 there are more sharp corners, so the average RD is lower. Increasing $r w$ creates more chaotic movements and more sharp changes of direction, which means that RD decreases.

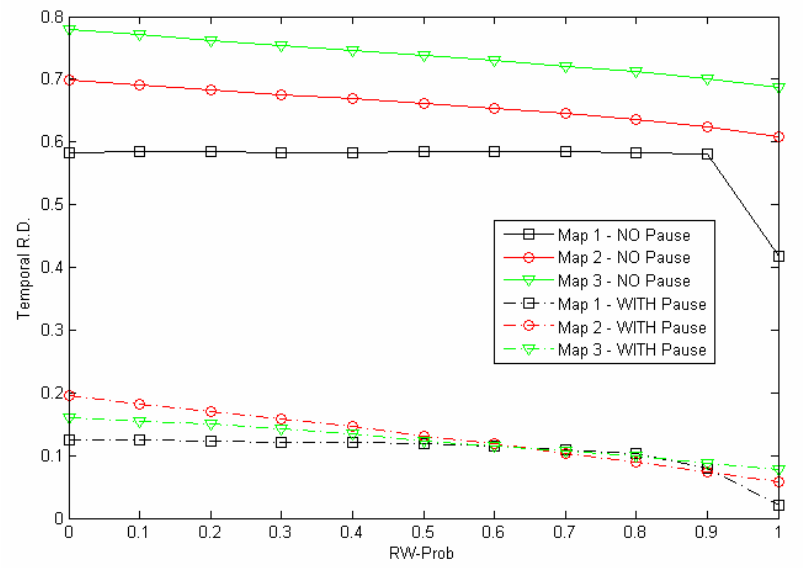

Figure 9 - Average temporal relative direction

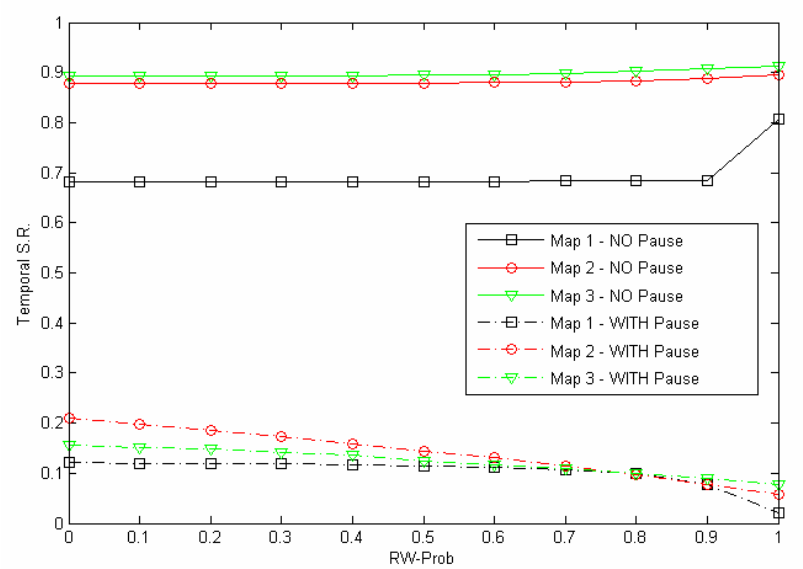

Figure 10 - Average temporal speed ratio

Since the speed of a node changes every step, when it moves in RWP, and every time it reaches an interconnection point of the graph, the average speed ratio (Figure 10) depends on how many times these events occur. When the nodes move along the pathways (low $r w$ ) the only factor is the complexity of the graphs. When they move in RWP, the speed ratio depends on the average number of steps, so the average duration of each. Next section is dedicated to the mathematical calculation of this parameter, and the comparison of analytical and simulation results.

\section{AVERAGE DURATION OF A STEP}

The purpose of this section is the calculation of the average duration of a step of a node. This parameter depends strongly on the simulation map and it is infeasible to calculate it for complex maps. However it is possible to study the case when the scenario is simple. The simplest case is when the simulation area is a rectangle with size $\left[X_{M}, Y_{M}\right]$ without any obstacles and the pathway graph is composed by one single destination vertex placed in the centre of the area. This case can represent the behaviour of a single room in a more complex scenario, from the moment a node arrives to the associated destination vertex till it leaves the room.

We want to calculate the expectation of the random variable $t$ :

$$
E(t)=E\left(\frac{d}{s}\right),
$$

where $d$ is the distance between the initial and the final position of the node and $s$ is the speed of the node.

Since $d$ and $s$ are independent,

$$
E(t)=E\left(\frac{1}{-} d\right)=E\left(\frac{1}{s}\right) E(d) .
$$

The speed $s$ is a random variable uniformly distributed between 1 and $5 \mathrm{~m} / \mathrm{s}$, so the variable $z \triangleq \frac{1}{s}$ has a distribution:

$$
f_{Z}(z)=\left.\frac{f_{S}(s)}{g^{\prime}(s)}\right|_{s=g^{-1}(z)}=\frac{1}{4 z^{2}} \quad \frac{1}{5} \leq z \leq 1
$$

Then the expectation is:

$$
E\left(\frac{1}{s}\right)=E(z)=\frac{1}{4} \ln (5)
$$

The distribution of the distance $d$ changes depending on the status of the movement. When the node starts the movement from the destination vertex (or when it moves to it) just one of the two points (initial or final position) has random coordinates. Instead, when the node is moving around the room in RWP movement, both of the two points are random.

Let's assume that the node starts moving from the destination vertex. It moves for a random distance $d$, that has a distribution $f_{D_{D V}}(d)$ (where DV stands for "destination vertex"). Then the node is going to choose a random destination with probability $r_{w}$ (RWP model), or it will come back to the destination vertex with probability $\left(1-r_{w}\right)$ (jumping into the graph). The probability of coming back to the destination vertex after $k$ steps is

$$
P_{k}=P(X=k)=\left(1-r_{w}\right) r_{w}{ }^{k} \quad k=0,1,2 \ldots,
$$

following a geometric distribution, which has a mean value

$$
E(X)=\frac{r_{w}}{1-r_{w}}
$$

Finally the node will come back to the destination vertex. 
So the node moves for $k$ times for a distance with distribution $f_{D_{R}}(d)$ (where $\mathrm{R}$ stands for "random") and 2 times (at the beginning and at the end) with distribution $f_{D_{D V}}(d)$.

Then the expectation of $d$ is:

$$
\begin{aligned}
E(d) & =\frac{1}{E(X)+2} \cdot\left[E(X) \cdot E_{R}(d)+2 E_{D V}(d)\right] \\
& =\frac{r_{w}}{2-r_{w}} E_{R}(d)+\frac{2-2 r_{w}}{2-r_{w}} E_{D V}(d)
\end{aligned}
$$

where $E_{R}(d)$ is the mean value of $f_{D_{R}}(d)$ and $E_{D V}(d)$ is the mean value of $f_{D_{D V}}(d)$

\subsection{Calculation of $E_{R}(d)$}

This parameter has been already calculated in [13]. While we have calculated the parameter different manner than in [13], we arrived at the same result, but omit our calculations due to space considerations and present only the result below.

$$
\begin{aligned}
& E_{R}(d)=\frac{1}{15}\left[\frac{X_{M}{ }^{3}}{Y_{M}{ }^{2}}+\frac{Y_{M}{ }^{3}}{X_{M}{ }^{2}}+\sqrt{X_{M}{ }^{2}+Y_{M}{ }^{2}}\left(3-\frac{X_{M}{ }^{2}}{Y_{M}{ }^{2}}-\frac{Y_{M}{ }^{2}}{X_{M}{ }^{2}}\right)\right]+ \\
& +\frac{1}{6}\left[\frac{Y_{M}{ }^{2}}{X_{M}} \cosh ^{-1}\left(\frac{\sqrt{X_{M}{ }^{2}+Y_{M}{ }^{2}}}{Y_{M}}\right)+\frac{X_{M}{ }^{2}}{Y_{M}} \cosh ^{-1}\left(\frac{\sqrt{X_{M}{ }^{2}+Y_{M}{ }^{2}}}{X_{M}}\right)\right]
\end{aligned}
$$

If $X_{M}=Y_{M}$ the result becomes

$$
E_{R}(d)=X_{M}\left[\frac{2+\sqrt{2}}{15}-\frac{1}{3} \ln (\sqrt{2}-1)\right] \simeq 0.5214 X_{M}
$$

\subsection{Calculation of $E_{D V}(d)$}

In this case the initial (or the final) position of the node is the destination vertex, positioned at $\left[\frac{X_{M}}{2}, \frac{X_{M}}{2}\right]$. The coordinates of the other position have a uniform distribution in $\left[0, X_{M}\right]$ for the $\mathrm{x}$-axis and $\left[0, Y_{M}\right]$ the $\mathrm{y}$-axis. So just one of the two positions is a random variable.

We want to calculate the distribution of the distance $d$, defined as:

$$
d \triangleq \sqrt{\left(x_{i}-x_{f}\right)^{2}+\left(y_{i}-y_{f}\right)^{2}}
$$

If we suppose that the final position is the destination vertex, we define

$$
a_{x} \triangleq x_{i}-\frac{X_{M}}{2}
$$

with the distribution:

$$
f_{A_{x}}\left(a_{x}\right)=\frac{1}{X_{M}} \operatorname{rect}\left(\frac{a_{x}}{X_{M}}\right)
$$

Then the distribution of $b_{x} \triangleq a_{x}^{2}$ is:

$$
f_{B_{x}}\left(b_{x}\right)=\frac{1}{X_{M} \sqrt{b_{x}}} \quad 0 \leq b_{x} \leq \frac{X_{M}^{2}}{4}
$$

Similarly, for the y-axis $b_{y}$ has a distribution:

$$
f_{B_{y}}\left(b_{y}\right)=\frac{1}{Y_{M} \sqrt{b_{y}}} \quad 0 \leq b_{y} \leq \frac{Y_{M}^{2}}{4}
$$

Then $c \triangleq\left(x_{i}-\frac{X_{M}}{2}\right)^{2}+\left(y_{i}-\frac{Y_{M}}{2}\right)^{2}=a_{x}^{2}+a_{y}^{2}=b_{x}+b_{y}$ has a distribution

$$
f_{C}(c)=\left\{\begin{array}{cc}
\frac{\pi}{X_{M} Y_{M}} & 0 \leq c<\frac{X_{M}^{2}}{4} \\
\frac{2}{X_{M} Y_{M}} \arcsin \left(\sqrt{\frac{X_{M}^{2}}{4 c}}\right) & \frac{X_{M}^{2}}{4} \leq c<\frac{Y_{M}^{2}}{4} \\
\frac{2}{X_{M} Y_{M}} \arcsin \left(\sqrt{\frac{X_{M}^{2}}{4 c}}\right)+ & \frac{Y_{M}^{2}}{4} \leq c \leq \frac{X_{M}^{2}+Y_{M}^{2}}{4} \\
\frac{2}{c-\frac{Y_{M}^{2}}{4}} & \left(\sqrt{\frac{1}{4}}\right)
\end{array}\right.
$$

The distribution of the distance $d \triangleq \sqrt{c}$ is (Figure 11):

$$
f_{D_{D V}}(d)=\left\{\begin{array}{cc}
\frac{2 \pi d}{X_{M} Y_{M}} & 0 \leq c<\frac{X_{M}}{2} \\
\frac{4 d}{X_{M} Y_{M}} \arcsin \left(\frac{X_{M}}{2 d}\right) & \frac{X_{M}}{2} \leq c<\frac{Y_{M}}{2} \\
\frac{4 d}{X_{M} Y_{M}} \arcsin \left(\frac{X_{M}}{2 d}\right)+ & \frac{Y_{M}}{2} \leq c \leq \frac{\sqrt{X_{M}^{2}+Y_{M}^{2}}}{2} \\
-\frac{4 d}{X_{M} Y_{M}} \arcsin \left(\sqrt{\frac{d^{2}-\frac{Y_{M}^{2}}{4}}{d^{2}}}\right) &
\end{array}\right.
$$




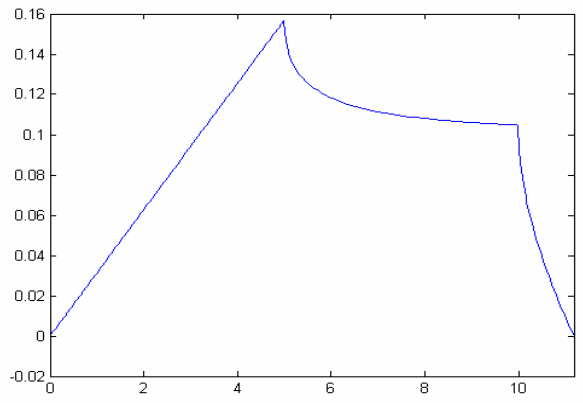

Figure $11-f_{D D V}(d)$ calculated with $X_{M}=10$ and $Y_{M}=20$

Finally, the expectation of the distance is:

$$
\begin{aligned}
E_{D V}(d)= & \frac{1}{6} \sqrt{X_{M}^{2}+Y_{M}^{2}}+\frac{1}{12} \frac{X_{M}^{2}}{Y_{M}} \operatorname{atgh}\left(\frac{\sqrt{X_{M}^{2}+Y_{M}^{2}}}{Y_{M}}\right)+ \\
& +\frac{1}{12} \frac{Y_{M}^{2}}{X_{M}}\left[\ln \left(\frac{\sqrt{X_{M}^{2}+Y_{M}^{2}}+X_{M}}{2}\right)-\ln \left(\frac{Y_{M}}{2}\right)\right]
\end{aligned}
$$

If $X_{M}=Y_{M}$ the expectation is

$$
E_{D V}(d)=X_{M}\left[\frac{\sqrt{2}}{6}+\frac{1}{8} \ln (\sqrt{2}+1)-\frac{1}{24} \ln (\sqrt{2}-1)\right] \simeq 0.3826 X_{M}
$$

\subsection{Final Results}

So, if the area of the simulation is a square $\left[X_{M}, X_{M}\right]$ and the destination vertex is positioned at $\left[\frac{X_{M}}{2}, \frac{X_{M}}{2}\right]$, we can calculate the average length of a step. Using the (3) and (4), the (2) becomes:

$$
\begin{aligned}
E(d) & \simeq \frac{r_{w}}{2-r_{w}} 0.5214 X_{M}+\frac{2-2 r_{w}}{2-r_{w}} 0.3826 X_{M} \\
& =X_{M}\left[\frac{0.7652-0.2438 r_{w}}{2-r_{w}}\right]
\end{aligned}
$$

and, using the (1), the average duration of one step is then:

$$
E(t)=E(d) E\left(\frac{1}{s}\right) \simeq X_{M}\left[\frac{0.7652-0.2438 r_{w}}{2-r_{w}}\right] \frac{\ln (5)}{4}
$$

Figure 12 and 13 show the comparison between analytical and simulation results in the calculation of $\mathrm{E}(\mathrm{d})$ and $\mathrm{E}(\mathrm{t})$.

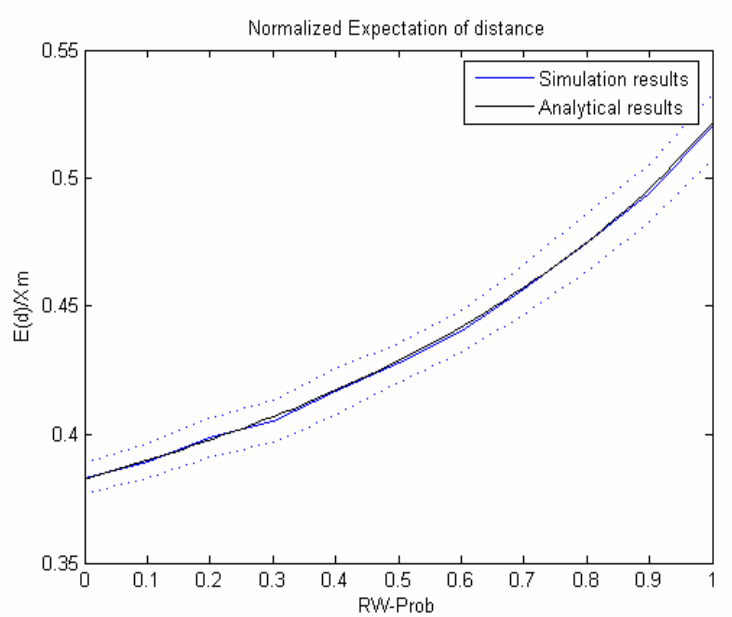

Figure 12 - Normalized E(d)

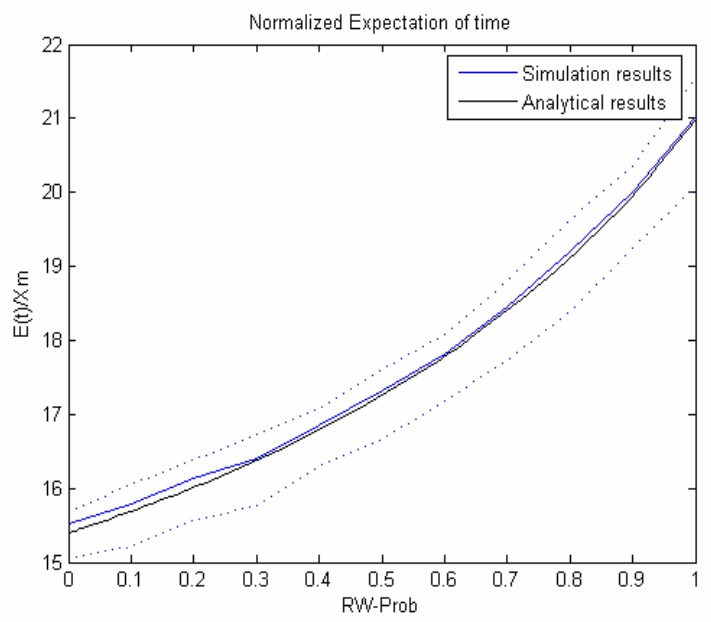

Figure 13 - Normalized E(t)

\section{CONCLUSIONS}

The paper describes a new mobility model based on one previously suggested in [4]. Our model enables the simulation of scenarios with obstacles, designed in particular for indoor environments (but easily applicable for outdoor environments too). The obstacles interfere with the movement of the nodes and reduce the signal transmissions.

The user specifies the map of the obstacles using an image file. Every colour of the image is associated to a material with specific properties (the signal attenuation degree and whether the material is crossable by the nodes). This allows the creation of complex scenarios and the study of environments very close to reality. The movement of the nodes is a hybrid of two sub-models: in the first sub-model it follows the rules of the Random Waypoint model, avoiding crossing obstacles; in the second sub-model the nodes move on a virtual graph defined by the user, choosing the shortest path to reach the destination vertex. During every step of the simulation the node chooses to use the first sub-model with a 
probability $r w$ or the second one with a probability 1-rw. The parameter $r w$ can depend on the sub-area in which the node is. For example, in reality, the movement of a node inside a room is more randomic than inside a corridor, where the people usually follow a linear path.

The use of only the first sub-model creates a too chaotic model. Every step is independent from the previous one. In real life a node does not move randomly, but following a specific path, especially for long distances. On the other hand, the use of only the second sub-model constrains the nodes to move on prefixed paths, without the possibility to move freely in the area. Both the solutions are thus not realistic. On the contrary, the mix of the two sub-models offers a more flexible and then realistic mobility model. Previous studies [2,3] showed that wireless networks simulations results are deeply dependent on the mobility model chosen. For that reason a model that imitates as much as possible the real world is important. Using too approximate models can bring results far from the reality.

There are many ways to extend this model. The first can be the use of a non-uniform distribution for destination selection. The destination can depend on the distance from the initial point and/or the specific node. For example only some nodes can be allowed to enter in certain areas. A second extension of the model can be the introduction of pauses at the entrances of the rooms. A third one can be an automatic sub-area division by the model (for example based on the virtual graph and the Voronoi algorithm [10]).

\section{REFERENCES}

[1] The Network Simulator (ns-2) http://www.isi.edu/nsnam/ns/

[2] Camp, T., Boleng, J., and Davies, V. 2002. A survey of mobility models for ad hoc network research. Wireless Communication \& Mobile Computing (WCMC): Special issue on Mobile Ad Hoc Networking: Research, Trends and Applications, 2 (5), 2002, pp. 483-502.

[3] Prabhakaran, P., and Sankar, R. 2006. Impact of Realistic Mobility Models on Wireless Networks Performance. In Wireless and Mobile Computing, Networking and Communications, WiMob’06, 19-21 June 2006, pp. 329334.

[4] Cavilla, A.L., Baron, G., Hart, T.E., Litty, L., and De Lara, E. 2004. Simplified simulation models for indoor MANET evaluation are not robust. In Proc. First Annual IEEE Communications Society Conference on Sensor and Ad Hoc Communications and Networks, IEEE SECON, October 2004, pp. 610-620.
[5] Bai, F., Sadagopan, N., and Helmy, A. 2003. IMPORTANT: a framework to systematically analyze the Impact of Mobility on Performance of Routing Protocols for Ad Hoc Networks. IEEE INFOCOM 2003, 30 March-3 April 2003, pp. 825835 vol. 2

[6] Hong, X., Gerla, M., Pei, G., and Chiang, C.C. 1999. A group mobility model for ad hoc wireless networks. In Proc. ACM/IEEE MSWIM'99, Seattle, WA, USA, August 1999, pp. 53-60.

[7] Bettstetter, C., and Wagner, C. 2002. The spatial node distribution of the random waypoint mobility model. In Proc. German Workshop on Mobile Ad Hoc Networks (WMAN), Ulm, Germany, Mar. 2002

[8] Mahajan, A., Potnis, N., Gopalan, K., and Wang, A. 2007. Modeling VANET Deployment in Urban Settings. In Proc. 10th ACM/IEEE International Symposium on Modeling, Analysis and Simulation of Wireless and Mobile Systems (MSWiM), Chania, Crete Island, Greece, October 2007.

[9] Jardosh, A., Belding-Royer, E.M., Almeroth, K.C., and Suri, S. 2003. Towards realistic mobility models for mobile ad hoc networks. MobiCom'03, September 2003, San Diego, California, USA, pp. 217-229.

[10] Okabe, A., Boots, B., Sugihara, K., and Chiu, S.N. 2000. Spatial Tessellations - Concepts and Applications of Voronoi Diagrams. 2nd edition. John Wiley, 2000.

[11] Tai Suk Kim, Jae Kyun Kwon, and Dan Keun Sung. 2000. Mobility modeling and traffic analysis in three-dimensional high-rise building environments. IEEE Transactions on Vehicular Technology, vol. 49, no. 5, September 2000, pp. 1633-1640.

[12] Dijkstra, E.W. A Note on Two Problems in Connection with Graphs. Numerische Mathematik, 1:269-271, 1959.

[13] Bettstetter, C., Hartenstein, H., and Perez-Costa, X. 2002. Stochastic Properties of the Random Waypoint Mobility Model: Epoch Length, Direction Distribution and Cell Change Rate. In Proceedings of the 5th ACM International Workshop on Modeling, Analysis, and Simulation of Wireless and Mobile SystemsMSWIM), Atlanta, GA, September 2002.

[14] Johnson, D.B., and Maltz, D.A. 1996. Dynamic source routing in ad hoc wireless networks. In Mobile Computing, Imielinski and Korth, Eds. Kluwer Academic Publishers, 1996, vol. 353.

[15] Royer, E., Melliar-Smith, P.M., and Moser, L. 2001. An analysis of the optimum node density for ad hoc mobile networks. In Proc. of the IEEE International Conference on Communications (ICC), 2001. 